\title{
Relationship of Lifestyle Habits with Sleep Quality among Japanese in Their 20s
}

\section{Tomohide Yagyu1, Kohei Akazawa², Minami Ebihara², Junta Ishikawa², Koichi Ouchi², Koji Kobayashi², Kyoko Nakazawa ${ }^{3}$}

\author{
${ }^{1}$ Department of Health Informatics, Suzuka University of Medical Science, Suzuka, Japan \\ ${ }^{2}$ Department of Medical Informatics, Niigata University Medical \& Dental Hospital, Niigata, Japan \\ ${ }^{3}$ Department of Medical Informatics and Statistics, Niigata University Graduate School of Medical \& Dental Sciences, \\ Niigata, Japan \\ Email: yagyu.tomohide@gmail.com
}

How to cite this paper: Yagyu, T., Akazawa, K., Ebihara, M., Ishikawa, J., Ouchi, K., Kobayashi, K. and Nakazawa, K. (2019) Relationship of Lifestyle Habits with Sleep Quality among Japanese in Their 20s. Health, 11, 1-8.

https://doi.org/10.4236/health.2019.111001

Received: December 11, 2018

Accepted: January 5, 2019

Published: January 8, 2019

Copyright (c) 2019 by author(s) and Scientific Research Publishing Inc. This work is licensed under the Creative Commons Attribution International License (CC BY 4.0).

http://creativecommons.org/licenses/by/4.0/

\begin{abstract}
The purpose of this study was to analyze the effects of lifestyle on sleep quality in young Japanese adults. A cross-sectional study was conducted on the web. We analyzed 637 eligible subjects (316 men and 321 women) aged 20 29 years. Information on diet and individual lifestyle characteristics, including sleep quality, which was assessed using the Pittsburgh Sleep Quality Index Japanese version (PSQI-J). Results were obtained by participants completing an online questionnaire. The participants were divided into two groups based on whether they were students ("student group") or not ("not student group"). The participants with a PSQI-J global score of 6 or higher were evaluated as low-sleep-quality. Logistic regression analyses were performed to evaluate associations of lifestyle habits with low-sleep-quality. Logistic regression analyses were applied for "student group" and "not student group". The results showed that days of eating out at dinner (OR: 1.11, CI: 1.00 - 1.22) and skipping dinner (OR: 1.21, CI: 1.05 - 1.42) were significant and independent predictors for low-sleep-quality among the "not student group". There were no significant and independent predictors for low-sleep-quality among "student group". Our results suggested that excessive intake by eating out and deficient intake by skipping dinner might lead to a high PSQI-J score. It is necessary for young people to receive education on the importance of cooking their own meals for traditional and daytime eating. The implication of this study lies in the fact that we have analyzed the effects of lifestyle habits on quality of sleep.
\end{abstract}

\section{Keywords}

Sleep Quality, Eating Out, Skipping Meal, Cross-Sectional Study, Pittsburgh Sleep Quality Index 


\section{Introduction}

Recently, the prevalence of short sleep duration has been increasing. $12 \%$ of US adults had reported sleep of $<6 \mathrm{~h}$ in 1998 [1]. However, during 2007-2010, up to $37.3 \%$ of US adults reported sleeping for $6 \mathrm{~h}$ or less [2]. According to a survey in Japan, the estimated rate of people sleeping for $<7$ hours was $31.8 \%$ [3].

Chen et al. highlighted how young adults who slept for $<7 \mathrm{~h}$ overnight were more likely to report low health-related quality of life than those sleeping for $\geq 7$ h overnight [4]. Previous studies have suggested that people with insufficient sleep syndrome have significantly higher depression scores than healthy sleepers, and that sleep problems are strongly associated with suicidal ideation and behavior [5] [6] [7] [8].

Several studies have been conducted to analyze factors which influence sleep. Skipping breakfast and excessive intake of staple foods at lunch and dinner are known to be associated with poor sleep-wake regularity [9]. In addition, other previous studies have suggested that sleep problems are associated with drinking [10], smoking [11], and exercise habit [12]. These are modifiable and common habits among students and others.

Our previous study was conducted to analyze the effects of eating out or skipping meals on the quality or quantity of sleep [13]. It suggested that excessive intake by eating out more than twice a week might lead to short sleep duration and high PSQI-J score [13]. The present study was conducted to analyze the effects of lifestyle on sleep quality in young Japanese adults.

\section{Materials and Methods}

\subsection{Study Design and Participants}

A cross-sectional study was conducted on the web in February 2017. Participants were recruited from a database that is administered by a Japanese private company specializing in questionnaire research. An online survey that was developed by a survey company, targeted individuals aged $20-29$ years without serious disease. Informed consent was obtained from all participants prior to enrollment. All 800 participants who accessed the survey web page responded to the questionnaire.

Exclusion criteria were: 1$)$ spends excessive hours in bed ( $\geq 16 \mathrm{~h} /$ day $[\mathrm{n}=65])$ and 2) responded with invalid answers to questionnaire items (e.g., selecting the same number on every question [ $\mathrm{n}=49])$; responded with answers that were inconsistent with those of other questions $(n=49)$. Finally, 637 participants (316 men and 321 women) were analyzed in this study.

\subsection{Questionnaire}

The questionnaire used in the present study consisted of three sections. Section 1 collected basic information on respondents, including age, sex, residential address, occupation, marriage status, and family structure. Section 2 comprised questions on lifestyle habit. Questions on diet were including frequency of skip- 
ping meals (breakfast, lunch, and dinner) per week. The survey respondents were also asked to provide average frequencies of visiting limited-service restaurants and full-service restaurants (breakfast, lunch, and dinner) per week. Other questions were comprised questions on days of exercise per week, current drinking and smoking (yes/no). Section 3 examined sleep quality, which was assessed by the Pittsburgh Sleep Quality Index Japanese version (PSQI-J) [14].

\subsection{Statistical Analysis}

The participants were divided into two groups based on whether they were students ("student group") or not ("not student group"). The cut-off point used was a 5.5 point in the PSQI-J global score because of its high sensitivity and specificity for primary insomnia 14. The participants with PSQI-J global score of 6 or higher were evaluated as low-sleep-quality.

Differences in diet and regular exercise between the participants evaluated as low sleep quality and others were analyzed by the Mann-Whitney U test. Differences in current drinking and smoking between the participants evaluated as low-sleep-quality and others were analyzed by the Fisher's exact test. The association of lifestyle habits, which were observed significant differences in the two groups' analysis, with low-sleep-quality prevalence, was investigated by logistic regression analysis. Kendall's tau coefficient was used to measure the ordinal association between each explanatory variable. Categorical variables were converted into dummy variables in logistic regression analyses. Logistic regression analyses were applied for "student group" and "not student group".

The R software package (version 3.4.1, 2017, The R Foundation for Statistical Computing) was used for all statistical analyses. All $\mathrm{p}$ values are two-tailed and those less than 0.05 were considered statistically significant.

\section{Results}

The basic characteristics of the participants included this study are shown in Table 1. Of these, 120 young adult participants were students, and 517 adult participants were not students. Significant differences were observed in age $(p<$ $0.001)$, sex $(p=0.004)$, occupation $(p<0.001)$, marriage status $(p<0.001)$ and sleep duration ( $p=0.016)$ between "student group" and "not student group".

There were significant differences in lifestyle habits between the participants evaluated as low-sleep-quality and others in the subjects with "student group" and "not student group". In "not student group", significant differences were evident in days of skipping dinner ( $p=0.038)$, eating out at morning $(p=0.016)$ and dinner $(p=0.003)$ (Table 2$)$. In "student group", significant differences were evident in days of exercise $(p=0.032)$ and skipping breakfast per week ( $p=$ 0.022 ) (Table 3 ). For the results obtained from measuring the ordinal association between these lifestyle habits, all values for the correlation coefficient were 0.02 to 0.35 . The association of these lifestyle habits with low-sleep-quality was investigated by logistic regression analysis. As shown in Table 4, days of eating 
Table 1. Baseline characteristics of the study subjects.

\begin{tabular}{|c|c|c|c|}
\hline & Student group & Not student group & $p$-value \\
\hline$N$ & 120 & 517 & \\
\hline Age (years) $\dagger$ & $22.0(20.0-23.0)$ & $27.0(25.0-28.0)$ & $<0.001 \S$ \\
\hline \multicolumn{4}{|l|}{ Sex $\ddagger$} \\
\hline Male & $74(61.7)$ & $242(46.8)$ & \\
\hline Female & $46(38.3)$ & $275(53.2)$ & 0.004 \\
\hline \multicolumn{4}{|l|}{ Occupation $\ddagger$} \\
\hline Full-time employee & $0(0)$ & $279(54.0)$ & \\
\hline Part time employee & $0(0)$ & $85(16.4)$ & \\
\hline Self-employed & $0(0)$ & $23(4.4)$ & \\
\hline Homemaker & $0(0)$ & $73(14.1)$ & \\
\hline Student & $120(100)$ & $0(0.0)$ & \\
\hline Unemployed & $0(0)$ & $57(11.0)$ & $<0.001$ g \\
\hline \multicolumn{4}{|l|}{ Marriage status $\ddagger$} \\
\hline Married & $2(1.7)$ & $152(29.4)$ & \\
\hline Unmarried & $110(91.7)$ & $317(61.3)$ & \\
\hline Divorced or widowed & $8(6.7)$ & $48(9.3)$ & $<0.001$ \\
\hline Days of exercise per week (days) $\dagger$ & $1.0(1.0-2.0)$ & $0.0(0.0-2.0)$ & $0.243 \S$ \\
\hline \multicolumn{4}{|l|}{ Current drinking $\ddagger$} \\
\hline Yes & $57(47.5)$ & $233(45.1)$ & \\
\hline No & $63(52.5)$ & $284(54.9)$ & 0.684 \\
\hline \multicolumn{4}{|l|}{ Current smoking $\ddagger$} \\
\hline Yes & $12(10.0)$ & $67(13.0)$ & \\
\hline No & $108(90.0)$ & $450(87.0)$ & 0.444 \\
\hline Sleep duration (hours) $\dagger$ & $7.0(6.0-8.0)$ & $6.8(6.0-7.1)$ & $0.016 \$$ \\
\hline PSQI-J score $\dagger$ & $4.0(3.0-7.0)$ & $5.0(3.0-7.0)$ & $0.261 \S$ \\
\hline
\end{tabular}

$\dagger:$ median (IQR); $\neq: n$ (percentage). Due to rounding, percentages may not total $100 \%$; $\$$ : $p$-values determined by using Mann-Whitney U test; $\mathbf{g}$ : $p$-values determined by using Fisher's exact test.

Table 2. Differences in lifestyle habits between the participants evaluated as low-sleep-quality and others in the subjects with "not student group".

\begin{tabular}{|c|c|c|c|}
\hline & \multirow{2}{*}{\multicolumn{2}{|c|}{$\begin{array}{cc}\text { With } & \text { Without } \\
\text { low-sleep-quality } & \text { low-sleep-quality }\end{array}$}} & \multirow{3}{*}{$p$-value } \\
\hline & & & \\
\hline & $n=196$ & $n=321$ & \\
\hline Sleep duration (hours) $\dagger$ & $6.1(6,5-7)$ & $7.0(7,6-8)$ & $<0.0001 \S$ \\
\hline PSQI-J score † & $8.3(7.5,7-10)$ & $3.3(3,2-4)$ & $<0.0001 \S$ \\
\hline Days of skipping breakfast (days/week) $\dagger$ & $1.7(0,0-3)$ & $1.6(0,0-2)$ & $0.293 \S$ \\
\hline Days of skipping lunch (days/week) $\dagger$ & $0.9(0,0-0)$ & $0.7(0,0-0)$ & $0.252 \S$ \\
\hline Days of skipping dinner (days/week) $\dagger$ & $0.5(0,0-0)$ & $0.2(0,0-0)$ & $0.038 \S$ \\
\hline Days of eating out at morning (days/week) $\dagger$ & $1.2(0,0-2)$ & $0.8(0,0-1)$ & $0.016 \S$ \\
\hline Days of eating out at lunch (days/week) $\dagger$ & $2.4(2,0-5)$ & $2.4(1,0-5)$ & $0.443 \S$ \\
\hline Days of eating out at dinner (days/week) $\dagger$ & $1.8(1,0-3)$ & $1.4(0,0-2)$ & $0.003 \$$ \\
\hline Days of exercise per week (days/week) $\dagger$ & $1.5(0,0-3)$ & $1.2(0,0-2)$ & $0.225 \$$ \\
\hline
\end{tabular}




\section{Continued}

\section{Current drinking $\ddagger$}

$\begin{array}{cccc}\text { Yes } & 86 & 147 & \\ \text { No } & 110 & 174 & 0.716\end{array}$

Current smoking $\ddagger$

\begin{tabular}{lcccc} 
Yes & 31 & 36 & \\
No & 165 & 285 & 0.139 \\
\hline
\end{tabular}

$\dagger:$ mean (median, IQR); $\neq: \mathrm{n} ; \mathfrak{\S}: p$-values determined by using Mann-Whitney $\mathrm{U}$ test; $\mathbf{g}: p$-values determined by using Fisher's exact test.

Table 3. Differences in lifestyle habits between the participants evaluated as low-sleep-quality and others in the subjects with "student group".

\begin{tabular}{|c|c|c|c|}
\hline & With & Without & \multirow{3}{*}{$p$-value } \\
\hline & \multicolumn{2}{|c|}{ low-sleep-quality low-sleep-quality } & \\
\hline & $n=44$ & $n=76$ & \\
\hline Sleep duration (hours) $\dagger$ & $6.2(6,5.4-7)$ & $7.3(7,7-8)$ & $<0.0001 \S$ \\
\hline PSQI-J score $\dagger$ & $8.2(8,6.8-9)$ & $3.0(3,2-4)$ & $<0.0001 \S$ \\
\hline Days of skipping breakfast (days/week) $\dagger$ & $2.8(2,0-6)$ & $1.7(0,0-3)$ & $0.022 \S$ \\
\hline Days of skipping lunch (days/week) $\dagger$ & $0.8(0,0-0.3)$ & $0.8(0,0-0)$ & $0.594 \S$ \\
\hline Days of skipping dinner (days/week) $\dagger$ & $0.6(0,0-0)$ & $0.6(0,0-0)$ & $0.44 \S$ \\
\hline Days of eating out at morning (days/week) $\dagger$ & $0.9(0,0-0.3)$ & $1.1(0,0-1)$ & $0.466 \S$ \\
\hline Days of eating out at lunch (days/week) $\dagger$ & $3.0(3,0-5)$ & $3.1(3,1-5)$ & $0.77 \S$ \\
\hline Days of eating out at dinner (days/week) $\dagger$ & $2.0(1,0-3)$ & $1.6(0,0-3)$ & $0.197 \S$ \\
\hline Days of exercise per week (days/week) $\dagger$ & $0.7(0,0-1)$ & $1.2(0,0-2)$ & $0.032 \S$ \\
\hline \multicolumn{4}{|l|}{ Current drinking $\ddagger$} \\
\hline Yes & 23 & 34 & \\
\hline No & 21 & 42 & $0.453 \mathrm{~g}$ \\
\hline \multicolumn{4}{|l|}{ Current smoking $\ddagger$} \\
\hline Yes & 6 & 6 & \\
\hline No & 38 & 70 & $0.353 g$ \\
\hline
\end{tabular}

$\dagger:$ mean (median, IQR); $\ddagger: \mathrm{n} ; \S: p$-values determined by using Mann-Whitney $\mathrm{U}$ test; $\mathbf{g}: p$-values determined by using Fisher's exact test.

Table 4. Odds ratios of lifestyle factors for high PSQI-J score among "not student group".

\begin{tabular}{cccc}
\hline & OR & $95 \% C I$ & $p$-value \\
\hline Days of eating out at morning & 1.07 & $0.96-1.18$ & 0.228 \\
Days of eating out at dinner & 1.11 & $1.00-1.22$ & 0.041 \\
Days of skipping dinner & 1.21 & $1.05-1.42$ & 0.012 \\
\hline
\end{tabular}

$p$-values determined by using logistic regression analysis.

out at dinner (OR: 1.11, CI: 1.00 - 1.22) and skipping dinner (OR: 1.21, CI: 1.05 1.42) were significant and independent predictors for low-sleep-quality among "not student group". As shown in Table 5, there were no significant and independent predictors for low-sleep-quality among "student group". 
Table 5. Odds ratios of lifestyle factors for high PSQI-J score among “student group".

\begin{tabular}{cccc}
\hline & OR & $95 \%$ CI & $p$-value \\
\hline Days of skipping breakfast & 1.14 & $0.99-1.32$ & 0.064 \\
Days of exercise per week & 0.82 & $0.61-1.06$ & 0.161 \\
\hline
\end{tabular}

$p$-values determined by using logistic regression analysis.

\section{Discussion}

In this study, the relationship between lifestyle habits (i.e., diet, regular exercise, current drinking and smoking) and quality of sleep was quantitatively evaluated based on a questionnaire survey.

Among adults who were not students, frequency of eating out at dinner was significant and independent predictor for high PSQI-J score. Nguyen and Powell showed that both fast-food and full-service restaurant food consumption among adults were associated with significant increases in energy, sugar, and saturated fat [15]. Further, according to other research, excessive intake of staple foods at lunch and dinner may be associated with poor sleep-wake regularity [9]. Given the findings of these previous studies and those of the current study, we considered excessive intake by eating out may lead to high PSQI-J score. In contrast, frequency of skipping dinner was also significant and independent predictor for high PSQI-J score. Kant and Graubard observed that short sleepers reported less breakfast and dinner consumption, and more frequent snacking [16]. So, we considered that deficient intake by skipping dinner may lead to high PSQI-J score. We think that it is necessary for young people to receive education on the importance of cooking their own meals for traditional and daytime eating. However, eating out could be due to having less time in the day to prepare meals, coinciding with increased stress and anxiety. This in turn also impacts sleep quality. Nguyen and Powell also showed that the effects of fast-food restaurant consumption were significantly larger for young (aged 20 - 34 years) compared with older adults [15]. It is also necessary to pay attention to dietary habits so that daily total energy intake is not increased by eating out.

Among students, significant differences were evident in frequencies of exercise and skipping breakfast per week between the participants evaluated as having insomnia and others. According to advanced research by Wang et al., regular exercise less than three times a week and skipping breakfast were associated with poor sleep quality [17]. Our findings did not contradict this report. But there were no significant and independent predictors for a high PSQI-J score. We think that a larger scale research will be needed.

The implication of this study lies in the fact that we analyzed the effects of lifestyle habits on quality of sleep. We assessed sleep quality by PSQI-J which could be quantitatively evaluated. And we used a cut-off point of 5.5 in the PSQI-J global score. Doi et al. showed that this cut-off point provided high estimations of sensitivity and specificity for primary insomnia, major depression, generalized anxiety disorder, and schizophrenia [14]. Therefore, we consider 
that our results showed relationship of lifestyle habits with not only sleep quality but also these psychiatric disorders.

However, this study also has several limitations. First, due to the nature of the cross-sectional study design, causal relationships between lifestyle habits and sleep quality should be interpreted with caution. Second, information on lifestyle habits and sleep quality was subjective, and can be considered as lacking objectivity. Further interventional studies by objective assessment of lifestyle habits and sleep quality will be needed. Our results show that days of eating out at dinner and skip-ping dinner were significant and independent predictors for low-sleep-quality among adults who were not student. Further studies between working time and diet habits will also be needed.

\section{Conclusion}

The present study was conducted to analyze the effects of lifestyle habits on sleep quality in young Japanese adults. Among adults who were not students, frequency of eating out at dinner and skipping dinner were significant and independent predictors for high PSQI-J score.

\section{Conflicts of Interest}

There is no conflict of interest to declare in this research.

\section{References}

[1] National Sleep Foundation (2002) 2002 "Sleep in America" Poll. http://sleepfoundation.org/sites/default/files/2002SleepInAmericaPoll.pdf

[2] Centers for Disease Control and Prevention (2007-2010) National Health and Nutrition Examination Survey. https://www.cdc.gov/nchs/nhanes/index.htm

[3] Ministry of Internal Affairs and Communications (2016) Survey on Time Use and Leisure Activities. https://www.e-stat.go.jp/en

[4] Chen, X., Gelaye, B. and Williams, M.A. (2014) Sleep Characteristics and Health-Related Quality of Life among a National Sample of American Young Adults: Assessment of Possible Health Disparities. Quality of Life Research, 23, 615-627. https://doi.org/10.1007/s11136-013-0475-9

[5] Morita, Y., Sasai-Sakuma, T., Asaoka, S. and Inoue, Y. (2015) Prevalence and Correlates of Insufficient Sleep Syndrome in Japanese Young Adults: A Web-Based Cross-Sectional Study. Journal of Clinical Sleep Medicine, 11, 1163-1169. https://doi.org/10.5664/jcsm.5092

[6] Spiegelhalder, K., Regen, W., Nanovska, S., Baglioni, C. and Riemann, D. (2013) Comorbid Sleep Disorders in Neuropsychiatric Disorders across the Life Cycle. Current Psychiatry Reports, 15, 364. https://doi.org/10.1007/s11920-013-0364-5

[7] McDonald, K.C., Saunders, K. and Geddes, J.R. (2017) Sleep Problems and Suicide Associated with Mood Instability in the Adult Psychiatric Morbidity Survey, 2007. Australian and New Zealand Journal of Psychiatry, 51, 822-828. https://doi.org/10.1177/0004867416687398

[8] Chu, C., Hom, M.A., Rogers, M.L., Stanley, I.H., Ringer-Moberg, F.B., Podlogar, M.C., Hirsch, J.K. and Joiner, T.E. (2017) Insomnia and Suicide-Related Behaviors: A Multi-Study Investigation of Thwarted Belongingness as a Distinct Explanatory 
Factor. Journal of Affect Disorders, 208, 153-162.

https://doi.org/10.1016/j.jad.2016.08.065

[9] Yamaguchi, M., Uemura, H., Katsuura-Kamano, S., Nakamoto, M., Hiyoshi, M., Takami, H., Sawachika, F., Juta, T. and Arisawa, K. (2013) Relationship of Dietary Factors and Habits with Sleep-Wake Regularity. Asia Pacific Journal of Clinical Nutrition, 22, 457-465. https://doi.org/10.6133/apjcn.2013.22.3.01

[10] Reen, E.V., Jenni, O.G. and Carskadon, M.A. (2006) Effects of Alcohol on Sleep and the Sleep Electroencephalogram in Healthy Young Women. Alcoholism, 30, 974-981.

[11] Zhang, L., Samet, J., Caffo, B. and Punjabi, N.M. (2006) Cigarette Smoking and Nocturnal Sleep Architecture. American Journal of Epidemiology, 164, 529-537. https://doi.org/10.1093/aje/kwj231

[12] Inoue, S., Yorifuji, T., Sugiyama, M., Ohta, T., Ishikawa-Takata, K. and Doi, H. (2013) Does Habitual Physical Activity Prevent Insomnia? A Cross-Sectional and Longitudinal Study of Elderly Japanese. Journal of Aging and Physical Activity, 21, 119-139. https://doi.org/10.1123/japa.21.2.119

[13] Yagyu, T., Akazawa, K., Ebihara, M., Ishikawa, J. and Koichi, O. (2018) Effect of Eating Out on Quality and Quantity of Sleep among Japanese in Their 20s. Health, 10, 1261-1269. https://doi.org/10.4236/health.2018.109097

[14] Doi, Y., Minowa, M., Uchiyama, M., Okawa, M., Kim, K., Shibui, K. and Kamei, Y. (2000) Psychometric Assessment of Subjective Sleep Quality Using the Japanese Version of the Pittsburgh Sleep Quality Index (PSQI-J) in Psychiatric Disordered and Control Subjects. Psychiatry Research, 97, 165-172.

https://doi.org/10.1016/S0165-1781(00)00232-8

[15] Nguyen, B.T. and Powell, L.M. (2014) The Impact of Restaurant Consumption among US Adults: Effects on Energy and Nutrient Intakes. Public Health Nutrition, 17, 2445-2452. https://doi.org/10.1017/S1368980014001153

[16] Kant, A.K. and Graubard, B.I. (2014) Association of Self-Reported Sleep Duration with Eating Behaviors of American Adults: NHANES 2005-2010. The American Journal of Clinical Nutrition, 100, 938-947. https://doi.org/10.3945/ajcn.114.085191

[17] Wang, L., Qin, P., Zhao, Y., Duan, S., Zhang, Q., Liu, Y., Hu, Y. and Sun, J. (2016) Prevalence and Risk Factors of Poor Sleep Quality among Inner Mongolia Medical University Students: A Cross-Sectional Survey. Psychiatry Research, 244, 243-248. https://doi.org/10.1016/j.psychres.2016.04.011 